\title{
Sur la valeur fourragère de quelques pâturages de la zone sous-Pyrénéenne de l'Aude
}

\author{
L. HEDIN et M. KERGUELEN \\ Laboratoire de Recherches sur les Plantes Fourragères I.N.R.A., Rouen, France
}

\begin{abstract}
Résumé
Dans cette étude, la composition botanique de quelques pâturages de la région pyrénéenne du département de l'Aude a été étudiée: la flore y varie notablement selon l'altitude et les types de sols, calcaires, schisteux ou granitiques, dont il est fourni quelques analyses granulométriques et chimiques.

Enfin, il est donné quelques indications sur le mode d'exploitation de ces pacages et les problèmes économiques de la région.
\end{abstract}

\section{Summary}

The fodder value of some grasslands of the cis-Pyrenean region of the Aude Department Studies on the botanical composition of some grasslands of the Pyrenean region of the Aude Department were undertaken; the flora there varies considerably according to the altitude and the types of soils: limy, schistose or granitic, of which some granulometric and chemical analyses are provided.

Finally some indications are given on the management of these grasslands and the economic problems of the region.

\section{Introduction}

Le but des études régionales que le Laboratoire de Recherches sur les Plantes Fourragères de Rouen consacre à la production fourragère en montagne est de faire l'inventaire des conditions actuelles d'exploitation, compte-tenu de l'état présent des pâturages et de préconiser les techniques qui nous paraissent indispensables pour leur amélioration. Pour que ces techniques aient quelque chance d'être adoptées, il faut qu'elles cadrent avec le niveau technique et les possibilités financières des agriculteurs.

Dans le présent exposé consacré à la zone sous-pyrénéenne du département de l'Aude, et particulièrement au pays de Sault, nous dirons tout d'abord quelles en sont les conditions géographiques particulières (climat et sol).

Nous verrons qu'il s'agit d'une zone de transition entre l'influence méditerranéenne et l'influence océanique. Nous donnerons ensuite quelques indications sur la structure agraire, l'importance de l'exode rural, le degré de la mécanisation.

L'étude botanique des pâturages constituera la part importante de ce travail, mais ces alpages ne sont qu'un élément de la production fourragère qui doit assurer l'alimentation du bétail pendant l'hivernage. Nous serons ainsi conduits à dire quelques mots des cultures d'herbe ou de fourrage réalisées par l'exploitant.

Reçu pour publication le 23 décembre 1964.

Neth. J. agric. Sci., Vol. 13 (1965) No. 2 (June) 


\section{Données géographiques}

\subsection{Cli matologie}

Il est difficile de préciser la climatologie de la zone visitée par manque d'informations météorologiques. La pluviométrie est très variable avec l'altitude, les conditions topographiques et l'exposition.

D'une façon générale, la région sous-pyrénéenne du département de l'Aude dont fait partie le pays de Sault est une zone pluvieuse où les chutes de pluie atteignent et dépassent 1 mètre par an à $800 \mathrm{~m}$ d'altitude. Une sécheresse relative se manifeste au cours des mois de juillet, d'août et de septembre. C'est la période des pluies d'orage. A Roquefeuil, on indique que des pluies très violentes ont parfois lieu au moment de la récolte des foins et que les eaux de ruissellement entrainent l'herbe fauchée. Quant à l'approvisionnement en eau de la végétation, il dépend également, ne l'oublions pas, de la nature du sol et du sous-sol; l'eau atmosphérique est plus ou moins énergiquement retenue de telle sorte qu'ainsi le climat peut être sensiblement modifié.

En l'absence de données météorologiques, on est conduit à examiner avec soin la composition botanique de la végétation pour se faire une opinion sur les conditions climatologiques qui prévalent à tel ou tel pâturage: l'importance relative des espèces montagnardes ou atlantiques peut nous renseigner quelque peu et nous ne manquerons pas de signaler ces caractéristiques pour nos divers relevés.

Quant à la nature des espèces cultivées qui peut parfois nous renseigner utilement, elle est, à cet égard, peu utile car le rendement des "variétés" utilisées dépend davantage de la technicité de l'agriculteur et de ses possibilités d'investissement. Un facteur important de la production fourragère est la durée de la végétation conditionnée en partie en zone montagneuse par l'altitude dont dépend la température moyenne.

En très grossière approximation, on peut considérer que les mois humides dont la température moyenne mensuelle est supérieure à $9^{\circ} \mathrm{C}$ sont des mois de végétation active, soit à $850 \mathrm{~m}$ environ, de début mai à mi-octobre.

En zone montagneuse, la neige recouvre le sol de façon plus ou moins continue de novembre à mars ou avril.

Notons que le rythme de végétation diffère également de ce qu'il est en plaine de zone atlantique. Le départ du pâturage y est d'une quinzaine de jours plus tardive et l'on n'observe pas un maximum de production fin mai ou début juin aussi marqué. Conduites en fauche, ces prairies donnent une récolte de foin moins importante par rapport au regain que dans les pays de basse plaine où le premier foin représente souvent les $3 / 4$ des récoltes suivantes. C'est tout au moins ce que l'on observe lorsque l'on examine les résultats de l'expérimentation très exhaústive menée par le G.N.I.S. ${ }^{1}$ fourrager depuis 1961 dans une trentaine de localités françaises (Cf. Ternant à $900 \mathrm{~m}$ dans le Puy-de-Dôme et Malouis dans l'Eure).

\subsection{Conditions de sol}

Elles sont assez variables. On y trouve des granites à l'intérieur de terrains calcaires, mais on rencontre également autour de granite des schistes métamorphiques durs, des grès calcaires et des dolomies foncées du Dévonien.

Dans la zone visitée, nous avons prélevé des sols riches en argile, décalcifiés, provenant sans doute de la décomposition de calcaires Aptiens = Urgoniens et des sols plus légers, contenant un pourcentage plus ou moins élevé en sables grossiers, d'origine granitique.

1 Groupement National Interprofessionnel des Semences. 
SUR LA VALEUR FOURRAGÈre DE QUELQUES PÂTURAGES DE LA ZONE SOUS-PYRÉNÉENNE...

Le TABLEAU 1 exige explications et commentaire. Nous commencerons par regrouper les prélèvements 1,1 bis, 1 ter, $3,4,7$ dont les $\mathrm{pH}-\mathrm{H}_{2} \mathrm{O}$ sont supérieurs à 5 , les taux d'argile relativevment élevés et les sables grossiers peu importants, les prélèvements $2,5,6$ correspondant à des sols granitiques, de $\mathrm{pH}-\mathrm{H}_{2} \mathrm{O}$ inférieur à 5 , beaucoup moins riches en argile, mais par contre renfermant un pourcentage important en sables grossiers.

Tableau 1. Analyses physiques et chimiques de sols de pâturages

\begin{tabular}{|c|c|c|c|c|c|c|c|c|c|}
\hline & \multicolumn{9}{|c|}{ Prélèvements } \\
\hline & 1 & 1 bis & 1 ter & 2 & 3 & 4 & 5 & 6 & 7 \\
\hline 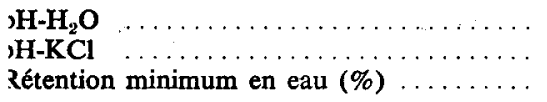 & $\begin{array}{r}5,5 \\
4,9 \\
75,6\end{array}$ & $\begin{array}{r}5,2 \\
5,0 \\
62,4\end{array}$ & $\begin{array}{r}6,5 \\
5,6 \\
139,1\end{array}$ & $\begin{array}{r}5,0 \\
4,1 \\
54,6\end{array}$ & $\begin{array}{r}7,0 \\
6,5 \\
94,6\end{array}$ & $\begin{array}{r}6,3 \\
5,4 \\
48,4\end{array}$ & $\begin{array}{r}4,7 \\
4,2 \\
78,1\end{array}$ & $\begin{array}{r}4,7 \\
4,0 \\
115,8\end{array}$ & $\begin{array}{r}6,1 \\
5,2 \\
70,1\end{array}$ \\
\hline \multicolumn{10}{|c|}{ Analyse physique en $\%$ de terresèche } \\
\hline 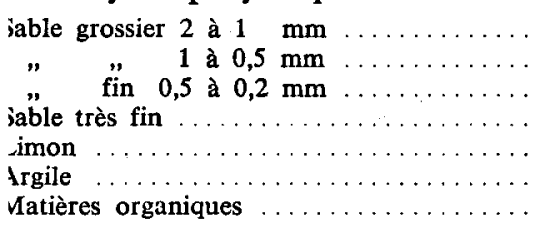 & $\begin{array}{r}1,3 \\
3,6 \\
8,4 \\
19,2 \\
20,9 \\
33,4 \\
13,2\end{array}$ & $\begin{array}{r}0,8 \\
3,4 \\
11,1 \\
14,2 \\
21,7 \\
36,7 \\
12,1\end{array}$ & $\begin{array}{r}0,09 \\
0,22 \\
0,09 \\
7,50 \\
24,30 \\
14,50 \\
53,30\end{array}$ & $\begin{array}{r}10,9 \\
12,6 \\
8,4 \\
16,0 \\
26,8 \\
18,0 \\
7,3\end{array}$ & $\begin{array}{r}0,15 \\
0,45 \\
2,00 \\
9,80 \\
37,60\end{array}$ & $\begin{array}{r}3,2 \\
4,1 \\
3,9 \\
1,8 \\
40,9 \\
30,0 \\
6.1\end{array}$ & $\begin{array}{r}10,8 \\
23,2 \\
11,2 \\
7,7 \\
18,9 \\
13,6 \\
14,6\end{array}$ & $\begin{array}{r}10,8 \\
12,3 \\
5,4 \\
12,7 \\
18,9\end{array}$ & $\begin{array}{r}1,9 \\
4,9 \\
6,9 \\
18,2 \\
33,4 \\
24,4 \\
10,3\end{array}$ \\
\hline \multicolumn{10}{|c|}{ Analysechimique en $\% 00$ de terresèche } \\
\hline 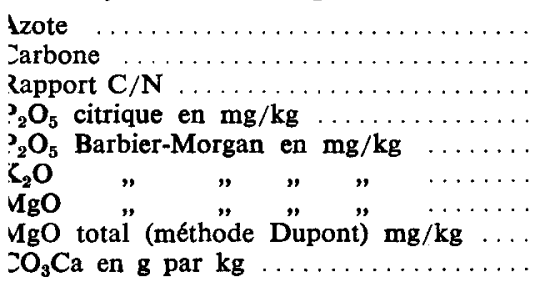 & $\begin{array}{r}5,3 \\
70,8 \\
13,2 \\
83,4 \\
5 \\
52 \\
133 \\
525\end{array}$ & $\begin{array}{r}4,5 \\
67,8 \\
15,0 \\
59,9 \\
6 \\
98 \\
434\end{array}$ & $\begin{array}{r}16,5 \\
389,4 \\
23,3 \\
225 \\
7 \\
62\end{array}$ & $\begin{array}{r}3,3 \\
41,3 \\
12,6 \\
133 \\
6 \\
117 \\
260 \\
344\end{array}$ & $\begin{array}{c}5,1 \\
64,9 \\
12,6 \\
156 \\
4 \\
31 \\
400 \\
2263 \\
7,7\end{array}$ & $\begin{array}{r}2,8 \\
35,4 \\
12,4 \\
71,0 \\
8,0 \\
31,0 \\
133 \\
525\end{array}$ & $\begin{array}{r}6,2 \\
88,5 \\
14,3 \\
165,7 \\
8 \\
176 \\
260 \\
434\end{array}$ & $\begin{array}{r}10,2 \\
159,3 \\
15,6 \\
170 \\
9 \\
184 \\
260 \\
326\end{array}$ & $\begin{array}{r}4,0 \\
47,2 \\
11,7 \\
251 \\
4 \\
29 \\
260 \\
471\end{array}$ \\
\hline \multicolumn{10}{|c|}{ Eléments échangeables en milliéquivalents par kg (Méthode MeHLich) } \\
\hline 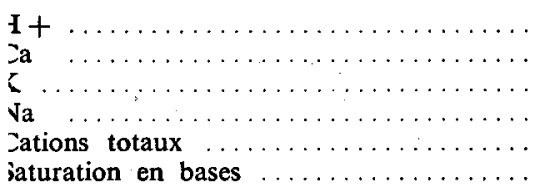 & $\begin{array}{c}172 \\
115 \\
3 \\
0,2 \\
321 \\
46\end{array}$ & $\begin{array}{c}195 \\
43 \\
3,5 \\
0,2 \\
261 \\
25\end{array}$ & $\begin{array}{c}96 \\
446 \\
3,3 \\
0,3 \\
626 \\
85\end{array}$ & $\begin{array}{r}169,4 \\
45,3 \\
5,0 \\
0,1 \\
231,7 \\
27\end{array}$ & $\begin{array}{r}66,3 \\
333,3 \\
1,9 \\
0,2 \\
436,9 \\
85\end{array}$ & $\begin{array}{r}24,3 \\
92,8 \\
2,4 \\
0,1 \\
232,3 \\
89\end{array}$ & $\begin{array}{r}244,2 \\
45,7 \\
5,7 \\
0,2 \\
31,6 \\
22\end{array}$ & $\begin{array}{r}343,5 \\
62,5 \\
6,7 \\
0,1 \\
474,2 \\
28\end{array}$ & $\begin{array}{r}164,5 \\
180,8 \\
1,6 \\
0,2 \\
405,9 \\
59\end{array}$ \\
\hline
\end{tabular}

1 , 1 bis et 1 ter sont des prélèvements effectués à Roquefeuil, dans les pâturages étudiés, 1 dans la zone travaillée, 1 bis dans la végétation prairiale non retournée. Le prélèvement 1 ter a été effectué dans une zone tourbeuse (mat.org. 53,3\%), qui a été cultivée en Maïs.

On notera une grande analogie entre 1 et 1 bis qui correspondent à des sols assez voisins l'un de l'autre. Le travail du sol semble avoir amélioré le rapport $\mathrm{C} / \mathrm{N}$ moins élevé pour 1 que 1 bis. Ce travail d'amendement du sol et l'apport d'amendement a également amélioré le $\mathrm{pH}$, la teneur en $\mathrm{P}_{2} \mathrm{O}_{5}$ citrique et la saturation en bases. Sols décalcifiés.

3 est un prélèvement fait dans les pâturages de la commune de Belcaire. C'est un 
sol qui renferme encore un peu de calcaire, à pH 7, remarquable par sa richesse en magnésie, particulièrement pauvre en $\mathrm{P}_{2} \mathrm{O}_{5}$. Bonne saturation en bases.

4 est un prélèvement fait dans une prairie temporaire à base de Dactyle, à Montplaisir, Commune d'Espezel. Si on compare cette terre à la précédente, on notera une teneur deux fois moindre en matière organique: c'est le résultat d'un travail habituel du sol. Rapport $\mathrm{C} / \mathrm{N}$ analogue, ce qui indiquerait que dans ces régions d'altitude, l'évolution de la matière organique est lente même dans des sols régulièrement cultivés. Bonne saturation en bases.

7 correspond à une prairie fauchée, dans la commune de Comus. Ces prairies fauchées reçoivent généralement du fumier. L'analyse ne fait pas apparaître un enrichissement du sol, si ce n'est une certaine richesse en $\mathrm{P}_{2} \mathrm{O}_{5}$ citrique.

2 est le sol qui correspond aux pâturages de la commune de Mazuby, dans le pays de Sault. Communaux.

5 est le sol des pâturages du Bousquet.

6 a été prélevé au Col de Jau.

Ces trois derniers sols, d'origine granitique, sont particulièrement riches en matières organiques à l'exception du $n^{0} 2$. Le rapport $\mathrm{C} / \mathrm{N}$ est élevé pour 5 et 6 . Du point de vue chimique, ces trois sols sont moyennement riches en $\mathrm{P}_{2} \mathrm{O}_{5}$ citrique et en $\mathrm{K}_{2} \mathrm{O}$ Barbier-Morgan.

\section{Le facteur humain}

Le climat et le sol sont des facteurs importants de la production fourragère, mais les conditions d'exploitation jouent également un rôle essentiel. Suivant la charge en animaux pâturant, les pacages se voient plus ou moins envahis d'arbrisseaux, de Fougères qui réduisent peu à peu les surfaces utilisables. Surpâturés, ils se dégradent rapidement par l'apparition d'espèces non consommées.

Voyons les conditions qui prévalent dans la zone qui dépend de l'inspection forestière de Quillan. Elle comprend les pâturages des cantons d'Axat et de Quillan qui s'étendent sur 7000 ha environ. Les forêts représentent 11.500 ha : 4000 ha de forêts domaniales, 3600 de forêts communales, 3900 appartenant à des particuliers. Forêts domaniales et forêts privées sont parcourues par des bovins.

Depuis une cinquantaine d'années, on note une diminution systématique de la population et par suite du nombre de bétail. Quelques chiffres préciseront la marche de cette dépopulation: pour les deux cantons, en 1901, le nombre d'habitants était de 22.000 ; il n'était plus que de 18.000 en 1920 , de 16.000 en 1936 , de 15.000 en 1954 . Cette diminution porte tout particulièrement sur la population rurale.

Le sous-pâturage favorise un envahissement de la végétation arborescente contre lequel les usagers luttent par le feu. Outre le drainage des tourbières et des mouillères, il est nécessaire d'utiliser le bulldozer pour combattre cette végétation.

L'ensemble de ces données conduit à poser le problème de l'orientation de l'équilibre sylvo-pastoral. Il semble qu'ici comme dans beaucoup d'autres cas, la solution à retenir dépend plus des facteurs socio-économiques que des possibilités d'amélioration du pâturage. Une forêt composée de Abies et de Fagus peut produire de 6 à $10 \mathrm{~m}^{3}$ par ha et par an de bois sur pied, commercialisable aux environs de 50 Frs.

L'hectare de forêt est susceptible de rapporter environ trois cents francs par an. Il est certain que si le pâturage donne une rente sensiblement moins élevée, cette dernière est acquise plus rapidement: en pâturage de montagne, l'hectare qui recevra 
des animaux pendant trois mois environ est rarement valorisé à plus d'une centaine de francs.

L'installation de prés-bois, qui est la solution adoptée en Espagne où l'importance des pâturages de montagne est très grande, est la technique à généraliser, notamment avec le mélèze en altitude $(1500 \mathrm{~m})$. La zone des pâturages améliorés serait celle qui se situe aux environs de $1000 \mathrm{~m}$. D'après les statistiques, dans le pays de Sault, les bois et forêts représentent $30 \%$ de la superficie, les pâturages et pacages $13 \%$ et les terres incultes $30 \%$.

L'affouragement d'hiver est produit en grande partie par l'exploitation même, il nous faut donner quelques indications sur sa structure. En presque totalité, il s'agit d'une exploitation familiale sans ouvrier agricole, de petite dimension. Dans le pays de Sault, si l'on excepte $15 \%$ environ de petites propriétés de moins d'un ha, les exploitations de moins de 8 ha représentent $55 \%$ du nombre total; $22 \%$ ont moins de 5 ha; $29 \%$ ont plus de 8 ha.

C'est dire les conditions précaires de la grande majorité des exploitations agricoles. Ajoutons les difficultés dûes à un parcellement très marqué, à un isolement dû à l'éloignement des centres urbains, à la médiocrité des chemins ruraux, de l'habitat. Non seulement les pertes de temps allongent la durée du travail, mais ce travail est pénible.

Quant à la structure même de l'exploitation, elle comprend outre quelques cultures de céréales et de pommes de terre, plus des deux tiers des surfaces consacrés soit aux fourrages assolés, soit aux prés de fauche fumés.

L'exploitation de M.O.... à Montplaisir, commune d'Espezel, est intéressante par l'effort d'intensification fourragère qui y a été réalisé. Elle s'étend sur 18 ha à $900 \mathrm{~m}$ d'altitude, sur sous-sol calcaire, décalcifié en surface. Sur cette superficie, 12 ha ont été convertis en prairies temporaires. La spéculation animale porte sur 200 ovins de la race de Lacaune élevés pour la production d'agneaux de boucherie.

La luzernière est constituée d'une variété sélectionnée à Castelnaudary. On en attend une première coupe de $5 \mathrm{~T}$ de foin et deux regains de 2,5 $\mathrm{T}$. Ici la mauvaise herbe de la luzerne est le Sonchus qui en gêne le développement.

Quant aux Graminées fourragères, M.O.... les associe à la luzerne: Dactyle-Luzerne, Fétuque des prés-Luzerne ou au Trèfle violet (Ray-grass d'Italie-Trèfle violet). Le Ray-grass d'Italie est préférable au Seigle fourrager. Quant à la Fléole des prés, elle a l'inconvénient d'être à ce point recherchée des moutons qu'elle disparaît après un pâturage. L'inconvénient des Graminées fourragères cultivées seules est la médiocrité de leur rendement en période sèche (rendement bien supérieur à celui des prairies naturelles).

La fertilisation annuelle est de 30 unités d'azote et 110 unités de $\mathrm{P}_{2} \mathrm{O}_{5}$ et $\mathrm{K}_{2} \mathrm{O}$ en scories potassiques.

Nous donnons par ailleurs une analyse de sol ( $n^{0} 4$, tableau 1) d'une culture de Dactyle et la composition botanique d'une prairie de fauche de M.O....

L'exploitation visitée n'est sans doute pas comparable à la moyenne communale qui est de 7 ha avec un parcellement excessif (parfois 30 parcelles par exploitation).

Ce qu'il faut retenir, c'est que l'exemple de M.O.... n'est pas suivi. Les essais de remembrement y échouent par suite de l'opposition de certains; le nombre des propriétaires est sensiblement plus important que celui des exploitants.

Bien que les charges à l'hectare soient nettement plus élevées chez M.O.... (200 ovins pour $18 \mathrm{ha}$ ), les conditions de vie de cet agriculteur demeurent précaires: une partie de son gain est utilisée pour le salaire du berger qui conduit le troupeau. 


\section{Les divers types de pâturages}

4.1. Méthodes utilisées pour l'analyse botanique

Pour les relevés botaniques quantitatifs, nous avons utilisé la "méthode de fréquences", préconisée par le Dr. D. M. DE VRIEs. Les résultats ont tous été traduits en \% relatifs de couverture végétale, en donnant l'indice 100 au total. Pour d'autres relevés, nous nous sommes contentés de noter les listes d'espèces présentes.

Nous avons classé, un peu arbitrairement, les espèces en graminées bonnes, moyennes et mauvaises - légumineuses - et plantes diverses: sans doute les notes de qualité à donner aux diverses espèces sont-elles susceptibles de varier selon les régions?

\subsection{Pât u rages de Roquefe u il (voir Tableau 2)}

Ce pâturage, géré par l'administration des Eaux et Forêts, est situé vers $900 \mathrm{~m}$ d'altitude, s'étend sur 250 ha et reçoit environ 500 bovins de la race Brune des Alpes de fin avril à octobre, pendant 150 jours environ.

A l'intérieur de ce pâturage qui se dégradait, avec envahissement de Pteridium aquilinum ${ }^{1}, 20$ ha ont été débroussaillés en 1962. Après emploi du bulldozer pour détruire la végétation arbustive (Prunus spinosa), on a procédé à un labour d'automne après apport, à l'hectare, de $1200 \mathrm{~kg}$ de scories, 300 unités de $\mathrm{K}_{2} \mathrm{O}$ et $1500 \mathrm{~kg}$ de chaux. Le semis a été effectué après discage, avec un mélange complexe $(10 \mathrm{~kg}$ de Lolium italicum, $10 \mathrm{~kg}$ de Dactylis glomerata variété Prairial, $10 \mathrm{~kg}$ de Festuca pratensis, $8 \mathrm{~kg}$ de Phleum pratense et $10 \mathrm{~kg}$ de Trifolium repens).

Un certain nombre d'observations s'imposent:

1. La préparation du sol au semis a été insuffisante.

2. Le mélange utilisé était trop complexe et coûteux, d'où l'importance des dépenses engagées, compte tenu des résultats obtenus.

Donnons maintenant 2 analyses botaniques, la première correspondant à l'état d'origine du pâturage, le seconde faite dans la partie "rénovée" 2 ans après le ressemis (TABLEAU 2).

On notera dans l'analyse $\mathrm{n}^{0} 2$ l'importance des bonnes graminées, notamment de la fléole (Phleum pratense), mais aussi le maintien de l'Agrostis tenuis, espèce médiocre et la réduction des plantes diverses. La faiblesse de la fertilisation, notamment azotée et l'exploitation peu rationnelle en sont sans doute la cause.

La composition botanique de la prairie originelle est assez banale pour une pelouse d'altitude, avec l'association Agrostis tenuis - Festuca ovina. Malgré l'acidité du sol (pH 5,2 - analyse 1bis, TABLEAU 1), nous sommes probablement sur terrain hétérogène avec touffes de Brachypodium pinnatum indiquant la présence de calcaire. Par contre Helianthemum ovatum, calcicole dans le nord de la France, croît sur terrains très variés en montagne.

Voici, au voisinage immédiat de ces pacages, un relevé des espèces rencontrées dans la tourbière du Pinot (analyse de sol $n^{0} 1$ ter, TABleau 1), en zone améliorée par le pâturage:

Festuca arundinacea ScHREB., Phleum pratense L., Festuca ovina L., Lolium perenne L., Holcus lanatus L., Molinia caerulea (L.) MoENCH.

Succisa praemorsa, Cirsium eriophorum (L.) Scop., Leontodon autumnalis, Potentilla anserina L., Juncus glaucus L., Plantago lanceolata, Veratrum album L., Gentiana lutea L., Genista anglica L., Juncus effusus L., Eupatorium cannabinum L.

' Orthographe prise dans: "Les quatre Flores de France" par P. FOURNIER. 
SUR LA VALEUR FOURRAGÈre DE QUELQUES PÂTURAGES DE LA ZONE SOUS-PYRÉNÉENNE ...

TABleau 2. Pâturage de Roquefeuil

\begin{tabular}{ll} 
Analyse \\
\hline 1 &
\end{tabular}

Bon nes graminé es

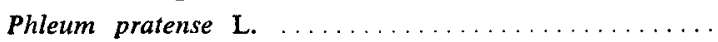

Festuca pratensis HUDS.

Dactylis glomerata $\mathbf{L}$.

Graminées moyennes

Poa pratensis L. s.l.

Trisetum flavescens (L.) P. BEAUV.

Graminées médiocres ou mauvaises

Agrostis tenuis SIBTH.

Festuca ovina L. sl.

Cynosurus cristatus $\mathrm{L}$.

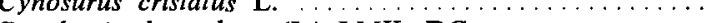

Danthonia decumbens (L.) LMK. DC. . . . . . . .

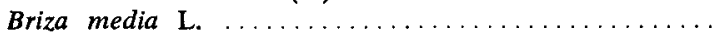

Brachypodium pinnatum (L.) P. BEAUV. . . . . . .

\section{Lé gumineuses utiles}

Trifolium repens $\mathrm{L}$.

Trifolium pratense $\mathbf{L}$. $\ldots \ldots \ldots \ldots \ldots \ldots \ldots \ldots \ldots \ldots$

Trifolium montanum $\mathbf{L}$.

Lotus corniculatus L.

\section{Plantes diverses}

Achillea millefolium L.

$\begin{array}{ll}0,8 & 7,0 \\ 4,6 & 1,7 \\ 2,3 & 1,7 \\ 1,5 & 1,7 \\ 1,5 & 5,2 \\ 4,6 & 1,7 \\ 0,8 & 3,4 \\ 5,4 & 3,4 \\ 0,8 & 3,4 \\ 1,5 & 5,2 \\ 3,1 & 1,7 \\ & 3,4 \\ 6,1 & 3,4\end{array}$

Plantago lanceolata $\mathrm{L} . \ldots \ldots \ldots \ldots \ldots \ldots \ldots \ldots$

Plantago media L. . . . . . . . . . . . . . . . .

Cerastium caespitosum GILIB. $\ldots \ldots \ldots \ldots \ldots \ldots \ldots$

Brunella vulgaris L. . . . . . . . . . . . . .

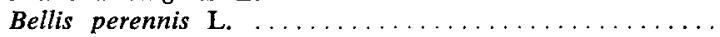

Potentilla tormentilla (L.) NECKER. . . . . . . . . .

Hypochaeris radicata $\mathrm{L}$.

Leontodon autumnalis $\mathrm{L}$.

Betonica officinalis $\mathrm{L}$.

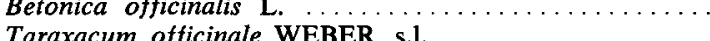

Helianthemum ovatum (VIV.) FOURNIER . . . . . . .

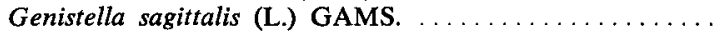

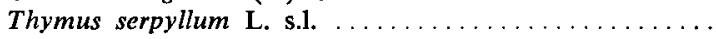

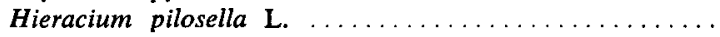



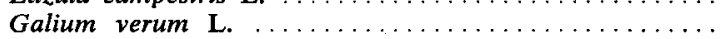

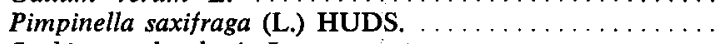

Scabiosa columbaria L. . . . . . . . . . . . . .

Succisa pratensis (GILIB.) ASCHERS. . . . . . . . .

Alchimilla vulgaris L. s.1. . . . . . . . . . . .

Carex ovalis GOOD. . . . . . . . . . . . . . . . .

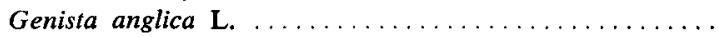

Genista tinctoria L.

Avec stade préforestier à Rhamnus frangula L., Prunus spinosa, Alnus glutinosa, Rubus sp.

Il s'agit d'une pelouse issue du dessèchement d'une tourbière neutre à hypnacées et Polytrichum commune. 


\subsection{Pâturages de Comus (voir tableau 3)}

Situés à $1250 \mathrm{~m}$ d'altitude, ils s'étendent sur 200 ha, pâturés par les animaux d'élevage. Nous sommes dans une zone en voie de dépopulation: si Comus comprend en 1963 environ 150 habitants (240 en 1938), avant 2 ans, il est probable qu'on ne comptera plus qu'une vingtaine d'exploitations.

Attenant aux zones de pâturages, nous observons diverses prairies de fauche typiques de l'étage montagnard du sapin (ici Abies pectinata DC. sous une race endémique particulière du "Sapin de l'Aude", qui semblerait voisin des Abies méditerranéens, tel A. normanniana).

Nous donnons dans le tableau 3 l'analyse botanique d'une de ces prairies fauchées: l'ensemble des prairies est situé sur sols calcaires, et la flore est d'assez bonne qualité.

Tableau 3. Prairie de fauche de Comus

Bonnes graminé es

Phleum pratense $\mathrm{L}$.

Lolium perenne $\mathrm{L} . \ldots \ldots \ldots \ldots \ldots \ldots \ldots \ldots \ldots \ldots$

Dactylis glomerata $\mathrm{L}$.

Graminé es moyennes

Trisetum flavescens (L.) P. BEAUV. . . . . . . . . . . .

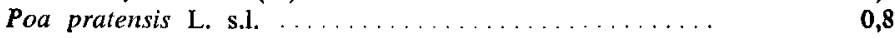

Graminées médiocres

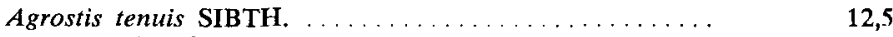

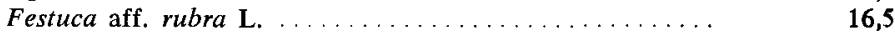

Bromus mollis L. . . . . . . . . . . . . . $1,6 \ldots \ldots . \ldots$

Légumine us es utiles

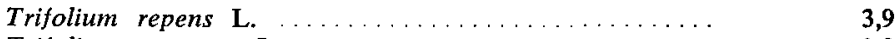

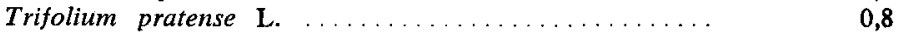

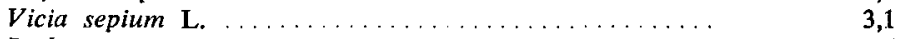

Lathyrus pratensis L. . . . . . . . . . . 1,6

Plantes diverses

Rhinanthus sp. ......................

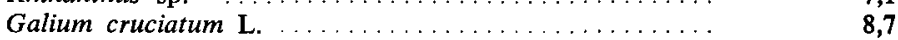

Chaerophyllum hirsuttim L. ...............

Veronica chamaedrys L. .................

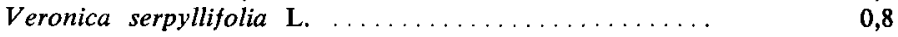

Taraxacum officinale WEBER. ................

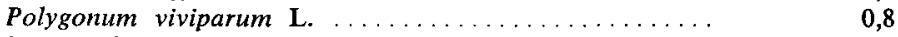

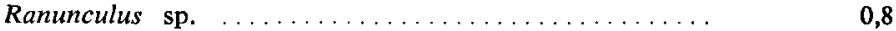

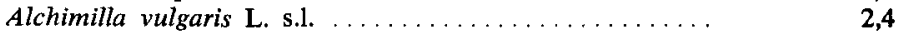

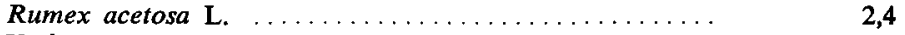

Viola cornuta L. . . . . . . . . . . . . . . . . . .

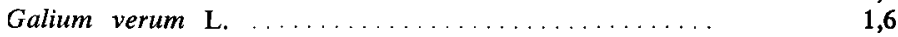

Conopodium majus LORET et GOUAN. $\ldots \ldots \ldots \ldots \ldots \ldots$
Centaurea montana L. $\ldots \ldots \ldots \ldots \ldots \ldots \ldots \ldots$

\subsection{Pâtu rage s du B o u s que t (voir tableau 4)}

D'une superficie de $508 \mathrm{ha}$, ils sont situés à $1650 \mathrm{~m}$ d'altitude sur sol granitique, en zone très arrosée: la pluviométrie annuelle est de l'ordre de $1500 \mathrm{~mm}$.

Le cheptel au pâturage est d'environ 362 bovins, pâturant du début de mai (23 mai en 1963) au 20 octobre: race bovine Gasconne. 
SUR LA VAlEUR FOURRAGère de QUELQUeS PÂTURAGES DE LA ZONE SOUS-PYRÉNÉENNE ...

Nous donnons dans le tableau 4 l'analyse botanique d'une partie de ces pâturages situés non loin des zones de rassemblement du troupeau: ces pâtures, à dominance d'Agrostis tenuis tendent au Nardetum par endroits, avec taches de landes, plus ou moins envahies de végétation arbustive, à Festuca eskia RAMOND., graminée très piquante et non consommable.

Tableau 4. Pâturage de Bousquet

Graminées moyennes

Poa pratensis L. s.1. 5,9

Poa trivialis $\mathbf{L}$.

Graminées médiocres ou sans valeur

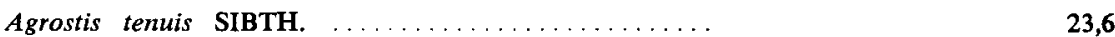

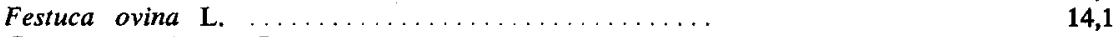

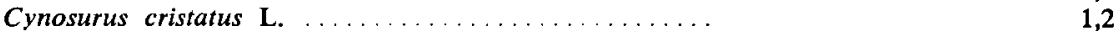

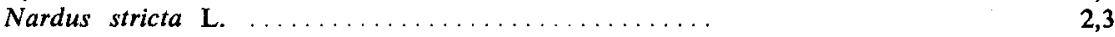

Légumineuses ut iles

Trifolium repens $\mathrm{L}$.

Plantes diverses

Plantago lanceolata L.

Ranunculus acer L.; sp. steveni . .............

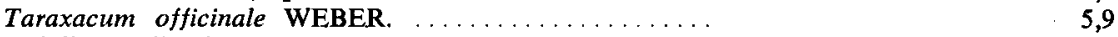

Achillea millefolium .....................

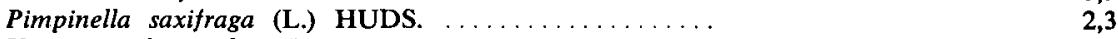

Veronica chamaedrys L. ...................

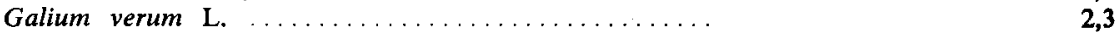

Carex flacca GOOD. (glauca Murr.) . . . . . . . . .

Carex ovalis GOOD. ......................

Conopodium majus LORET et GOUAN. .........

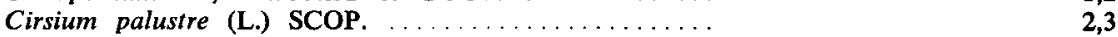

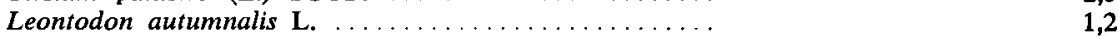



Cerastium caespitosum GILIB. . . . . . . . . 1,2

Mousses - sol nu

Tr a ces: Ranunculus pyrenaeus L., Gentiana pyrenaica L., Gentiana lutea L., Pedicularis palustris L., Potentilla tormentilla (L.) NECKER, Juncus effusus L., Hypericum maculatum CRANTZ., Succisa praemorsa (GILIB.) ASCHERS., Phleum alpinum L., Galium hercynicum WEIGEL., Juncus squarrosus L., Aconitum napellus L. s.1. - taches de Juniperus communis.

On se réfèrera à l'analyse de terre $\mathrm{n}^{0} 5$ (TABLEAU 1), indiquant une forte acidité, un rapport $\mathrm{C} / \mathrm{N}$ élevé, enfin le \% de sables grossiers dénotant le sol granitique.

A côté d'espèces endémiques pyrénéennes, comme Gentiana pyrenaica et d'espèces montagnardes ou arctiques-alpines comme Phleum alpinum, Juncus squarrosus, l'influence atlantique se fait sentir grâce à la présence de Conopodium majus, présente ici comme dans le Massif Central, mais absente des Alpes, absente aussi des zones pyrénéennes comme la Cerdagne, soustraites presque totalement aux influences océaniques.

\subsection{Pâturages de Belcaire (voir tableau 5)}

Ils sont situés à $1150 \mathrm{~m}$ d'altitude, sur calcaires urgo-aptiens relativement séchants. Une analyse d'une zone de ces pacages est donnée dans le TABLEAU 5.

Ce pâturage, relativement séchant, est toutefois d'assez bonne qualité pour la région, 
Tableau 5. Pâturages de Belcaire à $1150 \mathrm{~m}$ d'altitude

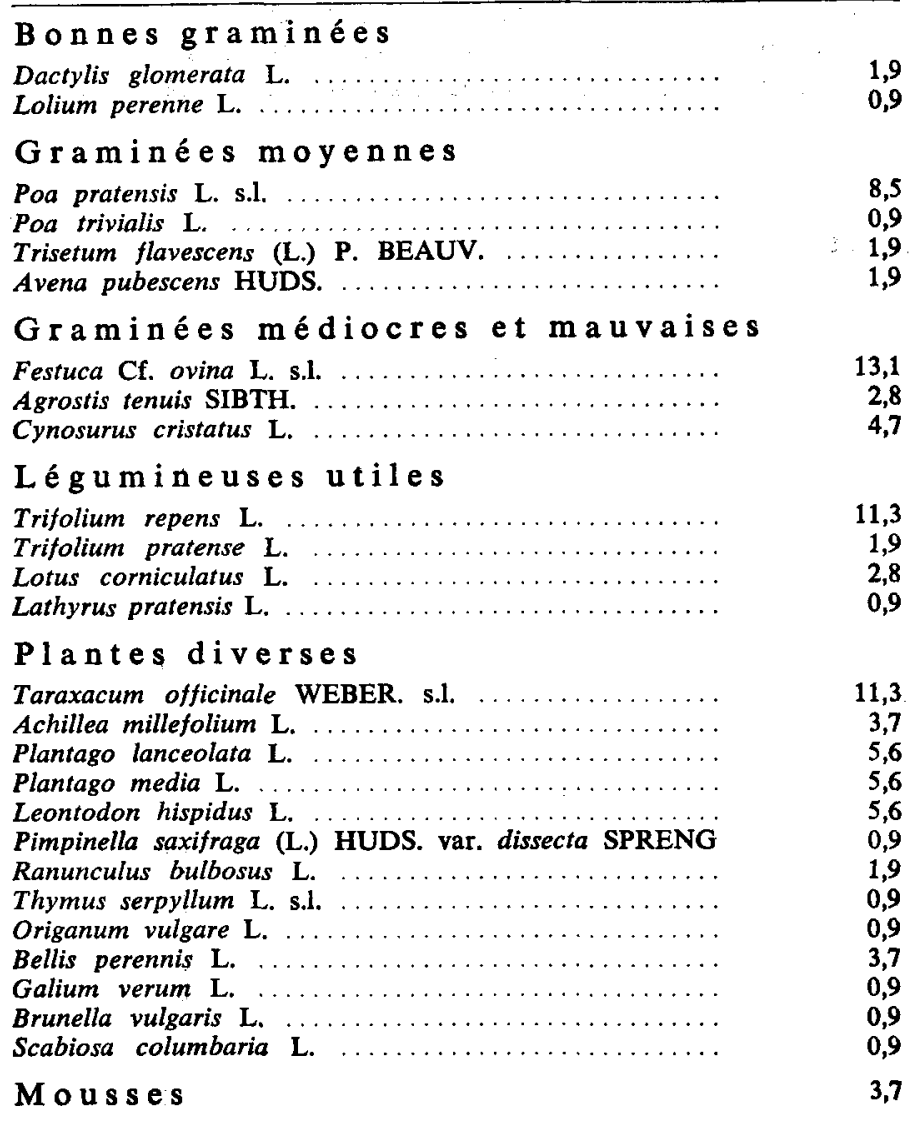

avec quelques graminées bonnes et moyennes, et un pourcentage relativement important de légumineuses, et d'espèces diverses consommables (Taraxacum - Plantago lanceolata).

4.6. Pâturages communaux de Mazuby (voir tableau 6)

D'une superficie de 395 ha, ils sont situés à $1150 \mathrm{~m}$ d'altitude, utilisés par 300 ovins et 140 bovins, dont 70 laitières, du 1er mai au 15 septembre.

Les animaux ont l'autorisation de pâturer dans certaines zones forestières où ils jouent un rôle utile pour la destruction des broussailles (Rubus).

Pâturages du Col de Ja u (voir Tableau 7)

Situés à $1513 \mathrm{~m}$ d'altitude sur sol granitique, ces pâturages forment un ensemble fort hétérogène, avec zones de gazon ras pâturé et zones sous pâturées à Cytisus purgans (L.) BENTH., espèce très "caractéristique du Massif Central et des Pyrénées et Juniperus communis. On y trouve aussi des combes à neige à Crocus albiflorus KrT.

L'analyse de terre permet les mêmes conclusions que l'échantillon provenant des pâtu- 
SUR LA VALEUR FOURRAGÈre DE QUELQUES PÂTURAGES DE LA ZONE SOUS-PYRÉNÉENNE ...

Tableau 6. Pâturages de Mazuby

Graminées moyennes

Poa pratensis $\mathrm{L}$.

Graminées médiocres et mauvaises

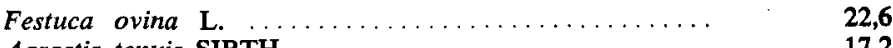

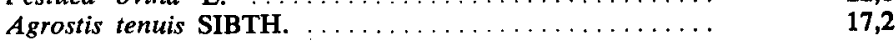

Légumineuses utiles

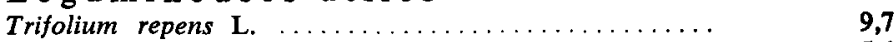

Lotus corniculatus L. ...................

Plantes diverses

Achillea millefolium L. ..................... 1,1

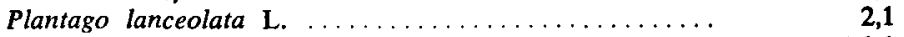

Plantago media L. ........................ $\quad 1,1$

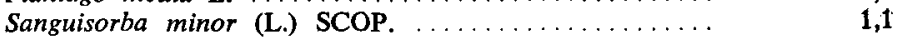

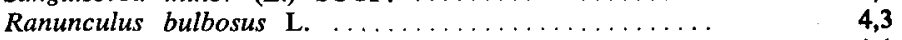

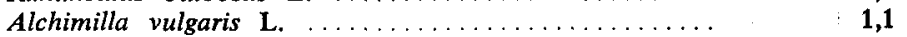

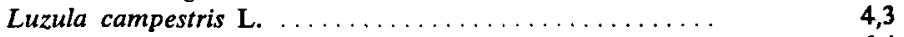

Galium verum L. . . . . . . . . .

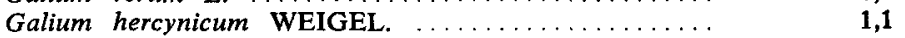

Veronica chamaedrys $\mathrm{L}$. . .................

Conopodium majus LORET et GOUAN. ............

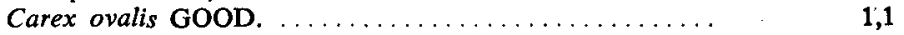

Hypericum montanum L. . . . . . . . $\ldots \ldots \ldots \ldots \ldots \quad \mathbf{1 , 1}$

Mousses 4,3

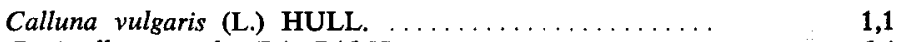

Genistella sagittalis (L) GAMS. . . . $\ldots \ldots \ldots \ldots \ldots \ldots \ldots \ldots, \quad 6,4$

Nota

Il s'agit là de pacages dégradés sur sols granitiques (analyse de terre $\mathrm{n}^{\circ} 2$ ), envahis d'arbustes, Juniperus, Prunus spinosa, Sarothamnus scoparius: type de la prairie à Agrostis - Festuca de médiocre valeur.

rages du Bousquet, eux aussi sur granite, à altitude comparable: col de Jau analyse $\mathrm{n}^{0} 7$ (TABLEAU 1).

Il s'agit là encore de pâturages pauvres, avec beaucoup d'espèces diverses sans intérêt. Notons la présence du Bromus erectus, fréquent dans les régions méridionales sur les sols granitiques, mais beaucoup plus lié aux calcaires dans le nord de la France.

\subsection{Pâturages de 1 a commune d'Espezel}

Situés vers $950 \mathrm{~m}$ d'altitude, la pluviométrie y est d'environ $1000 \mathrm{~mm}$, avec une sécheresse marquée en juillet: la plupart de ces pâturages sont encore du type Agrostis tenuis - Festuca ovina, avec envahissement fréquent de Pteridium aquilinum, Genistella sagittalis, Calluna vulgaris, Sarothamnus scoparius.

\section{Observations sur la composition botanique et la valeur économique des pâturages étudiés}

La composition botanique des pâturages étudiés dépend, comme l'on sait, à la fois de la nature du sol, de l'altitude et surtout du mode d'exploitation. La plupart d'entre eux sont des Agrostidaies, particulièrement sur les sols granitiques. Le Nard 
Tableau 7. Pâturage communal près de Jau à $1510-1520 \mathrm{~m}$ d'altitude

Graminées moyennes

Bromus erectus HUDS.

Graminé es médiocres ou sans valeur

Festuca ovina L.

Agrostis tenuis SIBTH.

Lé g u mineuses

Trifolium repens $\mathrm{L}$.

Trifolium alpinum $\mathbf{L}$.

\section{Plantes diverses}

Calluna vulgaris (L.) HULL.

Genista pilosa $\mathrm{L}$.

Helianthemum ovatum (VIV.) DUNAL s. sp. grandiflorum (LMK et DC.) ISSLER .....

Thymus nervosus GAY.

Selinum pyrenaeum (L.) GOUAN. $\ldots \ldots \ldots \ldots \ldots \ldots \ldots$

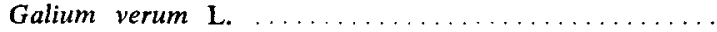

Galium cruciatum L.

Luzula campestris $\mathrm{L}$.

Achillea millefolium $\mathrm{L}$.

Serratula tintoria L. s. sp. macrocephala BERTOL. ...

Succisa praemorsa (GILIB.) ASCHERS. . . . . . . .

Crocus albiflorus KIT.

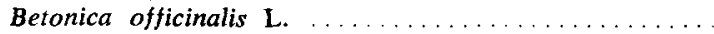

Carex ovalis GOOD.

Alchimilla alpina $\mathbf{L}$.

Potentilla tormentilla (L) NECKER

Pimpinella saxifraga (L.) HUDS.

\section{6,4}

20,4

9,6

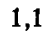

$+$

3,2

5,3

10,6

10,6
4,3
1,1

1,1

5,3

1,1

2,1

1,1

7,4

1,1

1,1

3,2

8,5

2,1

2,1

s'y rencontre sur granite dans les parties les plus dégradées et surtout en altitude avec Trifolium alpinum. C'est le cas notamment dans les pâturages du Bousquet à $1650 \mathrm{~m}$ d'altitude, au niveau limite de la forêt de Sapin; on y trouve des atlantiques comme Conopodium majus, Galium saxatile, des montagnardes comme Gentiana pyrenaica, Phleum alpinum, Ranunculus pyrenaicus, Aconitum napellus.

A Belcaire, sur sol calcaire, on voit apparaître Avena pubescens, Lotus corniculatus, Origanum vulgare, Scabiosa columbaria, Plantago media.

Lorsque le pâturage cède la place à une prairie de fauche fumée, on voit apparaître, comme à Comus, Trisetum flavescens, Poa pratensis, Dactylis glomerata, accompagnées de montagnardes, Centaurea montana, Viola cornuta.

Si nous essayons maintenant d'apprécier la valeur fourragère des pâturages étudiés, nous noterons qu'elle varie avec la nature du sol, granitique ou argileux, mais aussi avec l'altitude qui conditionne le nombre de jours de pâturage. La durée d'estivage est de 150 jours en moyenne au Bousquet, à $1650 \mathrm{~m}$ d'altitude, mais le début du pâturage qui se situe parfois au 10 mai, a eu lieu en 1963 au 23 mai. Sur 500 ha, on y entretient 365 bovins pour la production du veau de boucherie et du boeuf de travail. Evaluée en unités fourragères à l'hectare la production est de 7 à 800 unités. Les frais de gardiennage (1 vacher) s'élèvent pour la saison à 5000 Frs environ, soit 10 Frs à l'hectare. En très grossière approximation, le prix de revient de l'unité fourragère se situerait aux environs de 0,015 Frs pour les usagers de la commune. C'est évidemment le grand avantage de ce mode d'exploitation extensive qui permet au 
Bousquet des gains de poids appréciables des adultes et un accroissement substantiel de celui des jeunes.

Les pâturages sur granite de Mazuby $(1150 \mathrm{~m})$ en partie boisée, semblent avoir une production moindre de 6 à 700 U.F. Par contre, à Roquefeuil, $900 \mathrm{~m}$ d'altitude, sur sol argileux décalcifié, la production à l'hectare approcherait de 1500 U.F./ ha.

Il s'agit évidemment là d'une exploitation "minière" puisque l'on ne fait pas retour de ce qui est enlevé chaque année.

Le ressemis et une fertilisation correcte permettrait sans doute de doubler la productivité du pâturage et par suite la charge en bétail, tout en maintenant le prix de revient de l'U.F. à un prix très bas.

\section{Conclusions}

Pour parvenir à une meilleure alimentation du bétail accroissant sa rentabilité, il est nécessaire d'améliorer le rendement des pâturages pendant l'estivage et d'augmenter les ressources fourragères (fourrages assolés, prairies temporaires) pour la période hivernale.

1. Sur l'équilibre sylvo-pastoral et l'exploitation des pât u ra ges

L'exploitation extensive des pâturages communaux, nous l'avons vu, a l'avantage de fournir aux animaux une alimentation fourragère à bon compte. Mais l'extensification n'implique pas que l'on doive négliger de réaliser une exploitation rationnelle. Nous retrouvons ici les règles ordinaires de la bonne exploitation.

a. Réaliser un bon équilibre entre les possibilités du pâturage et le chargement en bétail. Ce qui caractérise une mauvaise exploitation, c'est la proximité de zones sous-pâturées où se développe un stade pré-forestier (Fougères, Calluna, Juniperus, etc. ..) et de zones surpâturées où les bonnes Graminées sont remplacées par Nardus stricta, Genistella sagitallis, Ononis spinosa. La rotation méthodique des pâturages, cloisonnés ou non, s'impose ici comme ailleurs.

b. Fertilisation. Il est certain qu'elle est plus coûteuse qu'en région de plaine, mais partout où la flore est de bonne qualité, l'apport de scories potassiques et d'azote augmentera sensiblement les rendements.

c. Installation de prairies temporaires. Là où une flore dégradée ne permettrait pas une rentabilité suffisante des engrais et où les conditions de sol permettent le labour, le retournement de la végétation naturelle et le ressemis s'imposent.

Sans doute cette implantation nouvelle implique-t-elle des investissements et du travail, sol bien préparé, tassé en surface. C'est la raison pour laquelle il importe de réduire les dépenses de semences, en utilisant des mélanges simples (une Graminée, une Légumineuse): semer du Fromental (Arrhenaterum elatius) dans une prairie qui sera pâturée est un acte fait en pure perte.

Les nombreuses mises au point qui ont été réalisées pour les terres cultivées de plaine sont valables ici. De toutes façons, pour se faire une opinion même approximative du comportement de telle ou telle espèce de Graminée, il est préférable de la semer isolément que de la semer dans un mélange complexe.

La solution très tentante de semer directement après suppression du gazon naturel (paraquat) apparait actuellement encore trop coûteuse.

Il ne faut pas oublier l'importance de la fertilisation sur un ressemis. Une végétation comprenant des espèces plus productives exige des engrais de fonds (phospho-potas- 
SUR LA VALEUR FOURRAGÈre de QUELQUeS PÂTURAGES DE LA ZONE SOUS-PYRÉNÉENNE...

siques) et une fertilisation azotée, notamment au printemps, lorsque la nitrification est peu intense.

Nous souhaiterions ici voir s'installer la technique du "complementary grazing" que nous avons vu réaliser en Ecosse. Il consiste à établir une prairie temporaire sur un sol facile à travailler, les animaux consommant rapidement le matin de l'herbe jeune, riche en protéine. L'après-midi ils sont en mesure de pâturer une herbe plus grossière et même une végétation semi-ligneuse. On note un transfert de fertilité de la prairie temporaire établie au centre du pâturage. La surface du ressemis par rapport à l'ensemble de la superficie du pacage peut être de l'ordre de 10 à $15 \%$.

Dans les conditions du Pays de Sault les pâturages et pacages représentent $13 \%$ de la surface et les terres incultes, c'est à-dire les anciennes cultures et pâturages abandonnés $30 \%$.

Dans la recherche d'un équilibre sylvo-pastoral rationnel, il semble que les surfaces fourragères doivent de préférence être localisées sur les zones abandonnées par la culture. Les parties envahies par un stade pré-forestier auraient intérêt sans doute à être reboisées.

L'installation de pré-bois est une solution qui nous apparait avantageuse tant pour l'amélioration du pâturage que pour la protection des animaux pendant les périodes de pluie et de brouillard. Il faut reconnaitre qu'elle n'a pas toujours l'agrément des forestiers soucieux trop exclusivement de la production ligneuse.

Certains zootechniciens pensent que dans les conditions économiques actuelles l'élevage et la production de viande auraient grand intérêt à se déplacer vers les zones montagneuses où le coût de l'alimentation fourragère est relativement bas. $\mathrm{Ce}$ point de vue implique une extension des pâturages de montagne améliorés.

En terminant nous rappellerons que les cultivateurs de la commune ont intérêt à faire pâturer le plus possible leurs estives par des animaux de la commune plutôt que d'en faire bénéficier les propriétaires d'animaux transhumants.

2. Intensification de la production fourragère des exploitations

Les animaux ne demeurent que 5 mois environ sur les pâturages de montagne. Ils consomment pendant 7 mois des fourrages de l'exploitation.

L'accroissement du cheptel des habitants de la commune implique un développement des cultures fourragères de l'exploitation. Pour que ce développement soit réalisé avec le moins d'erreurs techniques possibles, un certain nombre d'expérimentations s'impose, - recherche des types de luzerne les plus intéressants - essais sur les associations de luzerne et de graminées - appréciation du mérite réciproque des divers types de Dactyle - intérêt de la Fléole, du Ray-grass d'Italie.

En extrême synthèse, si nous pensons que des essais simples mais interprétables s'imposent pour accrôtre la production fourragère aussi bien des pâturages que de l'exploitation, nous n'ignorons pas que des crédits d'investissement sont nécessaires tout autant que la formation technique de ceux d'entre les jeunes qui sont attachés à leur région. 